\title{
A case of advanced renal cell carcinoma with peritoneal carcinomatosis responding to sorafenib therapy
}

\author{
Akihisa Yao $\cdot$ Hideto Iwamoto $\cdot$ Shuichi Morizane \\ Tadahiro Isoyama • Takehiro Sejima • \\ Atsushi Takenaka
}

Received: 7 September 2011 / Accepted: 20 April 2012

(C) The Japan Society of Clinical Oncology 2012

\begin{abstract}
Peritoneal carcinomatosis is a relatively rare presentation of renal cell carcinoma. We report the case of an advanced renal cell carcinoma patient with peritoneal carcinomatosis who received sorafenib and achieved a dramatic and ongoing response in the metastatic lesion. After 6 months of sorafenib administration, despite a CT scan showing a stable left renal mass, the patient had marked shrinkage of the peritoneal nodules and ascites had disappeared. To our knowledge, this is the first report showing therapeutic activity of sorafenib against peritoneal carcinomatosis in a patient with renal cell carcinoma.
\end{abstract}

Keywords Renal cancer - Peritoneal carcinomatosis .

Sorafenib

\section{Introduction}

Renal cell carcinoma (RCC), which accounts for 2-3\% of all adult malignant neoplasm, has a tendency to metastasize. The frequent sites of metastases are the lung, lymph nodes, liver, and bone. Intraperitoneal metastatic spread of RCC involving mesentery and omentum is very uncommon [1].

Sorafenib is an orally available inhibitor of multiple receptor tyrosine kinases, including vascular endothelial growth factor receptor (VEGF), platelet-derived growth factor receptor (PDGF), and others, with direct antitumor

A. Yao $(\bowtie) \cdot$ H. Iwamoto $\cdot$ S. Morizane $\cdot$ T. Isoyama .

T. Sejima $\cdot$ A. Takenaka

Division of Urology, Department of Surgery,

Faculty of Medicine, Tottori University,

86 Nishi-cho, Yonago 683-8504, Japan

e-mail: ayao@med.tottori-u.ac.jp and antiangiogenic activity. On the basis of impressive outcomes in several clinical trials, sorafenib has been approved worldwide for treatment of RCC, and such tyrosine kinase inhibitors induce a high partial response rate at metastatic sites [2]. Herein, we report the case of an advanced RCC patient with peritoneal carcinomatosis who received sorafenib and achieved a dramatic and ongoing response in the metastatic lesion.

\section{Case report}

In March 2010, a 73-year-old man with a 5.5-cm left renal mass was referred to our institution. The CT scan also showed innumerable soft tissue implants throughout the peritoneum, a large amount of ascites, and renal hilar lymph nodes (Fig. 1). Karnofsky performance score was judged to be $80 \%$. The laboratory test results were hemoglobin $10.1 \mathrm{~g} / \mathrm{dl}$, lactate dehydrogenase $215 \mathrm{U} / \mathrm{l}$, corrected calcium $5.1 \mathrm{mg} / \mathrm{dl}$, and Memorial Sloan-Kettering Cancer Center classification was an intermediate risk. Although serum liver-associated enzymes, creatinine, and blood urea nitrogen were within normal limits, laboratory evaluation included total protein $7.8 \mathrm{mg} / \mathrm{dl}$ and albumin $1.7 \mathrm{~g} / \mathrm{dl}$. Diagnostic paracentesis was performed, which revealed visibly bloody peritoneal fluid. The differential cell count was $400 / \mathrm{mm}^{3}$ : neutrophils $63 \%$, monocytes $31 \%$, and lymphocytes $6 \%$, with a specific gravity of 1.028 , a total protein of $4.4 \mathrm{~g} / \mathrm{dl}$, an albumin of $0.7 \mathrm{~g} / \mathrm{dl}$, giving a serumascites albumin gradient (SAAG) of $1.0 \mathrm{~g} / \mathrm{l}$, which was suggestive of non-portal hypertension ascites. However, as this paracentesis was negative for malignant cells by cytologic evaluation, we performed a percutaneous, CT-guided biopsy of the left renal mass which revealed the histology of clear cell renal cell carcinoma (RCC). 

showed a a $5.5-\mathrm{cm}$ left renal mass (black arrow) and a large amount of ascites and $\mathbf{b}$ soft tissue implants throughout the peritoneum (white arrows)
Fig. 1 Pretreatment CT scan
Fig. 2 After 3 months of sorafenib therapy, a CT scan showed a a stable left renal mass (black arrow) and $\mathbf{b}$ the peritoneal nodules (white arrows); however, the ascites had disappeared
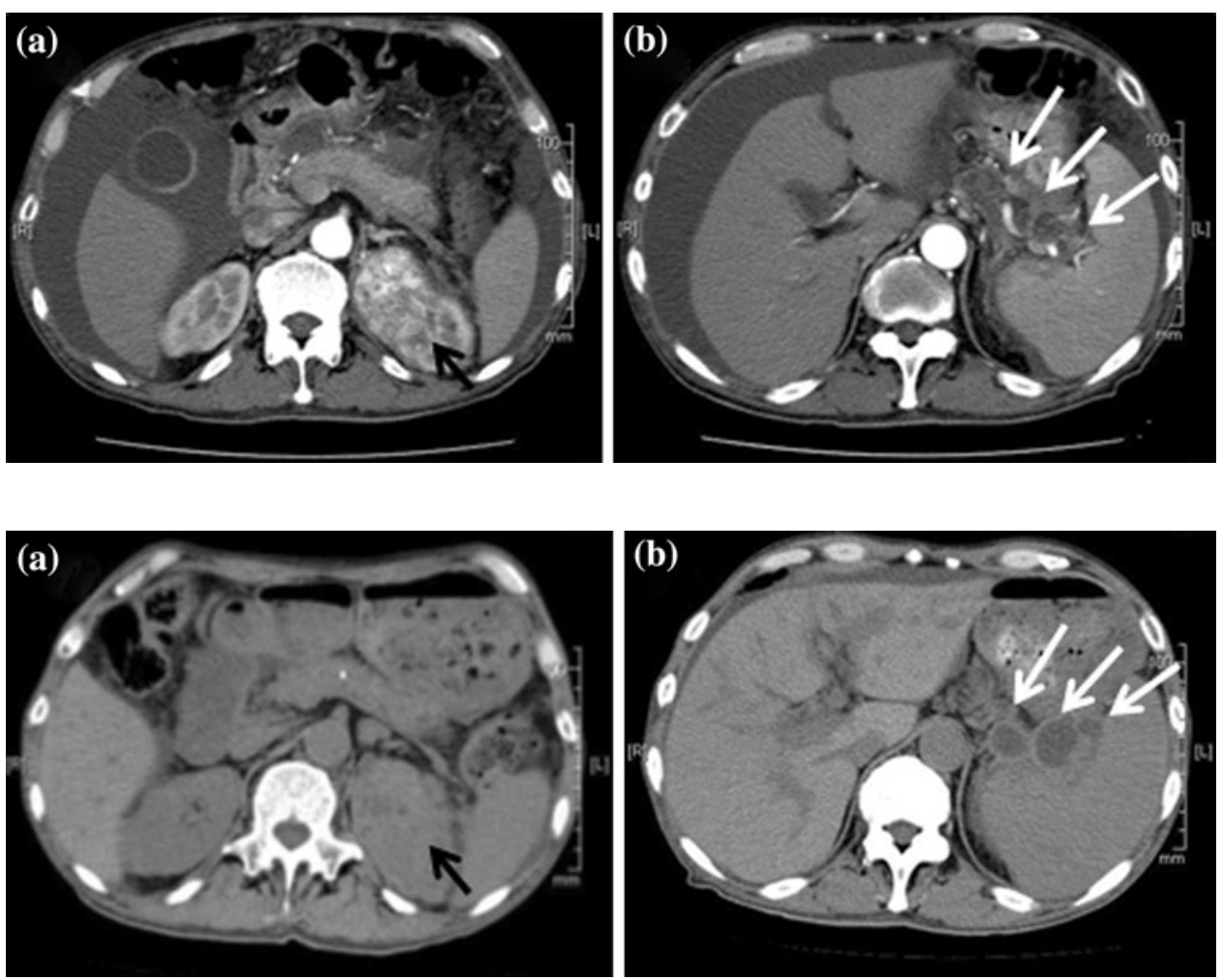

Fig. 3 After 6 months of sorafenib therapy, a CT scan showed a stable left renal mass (black arrow),

disappearance of the ascites, and b $95 \%$ reduction in the peritoneal nodules (white arrows)
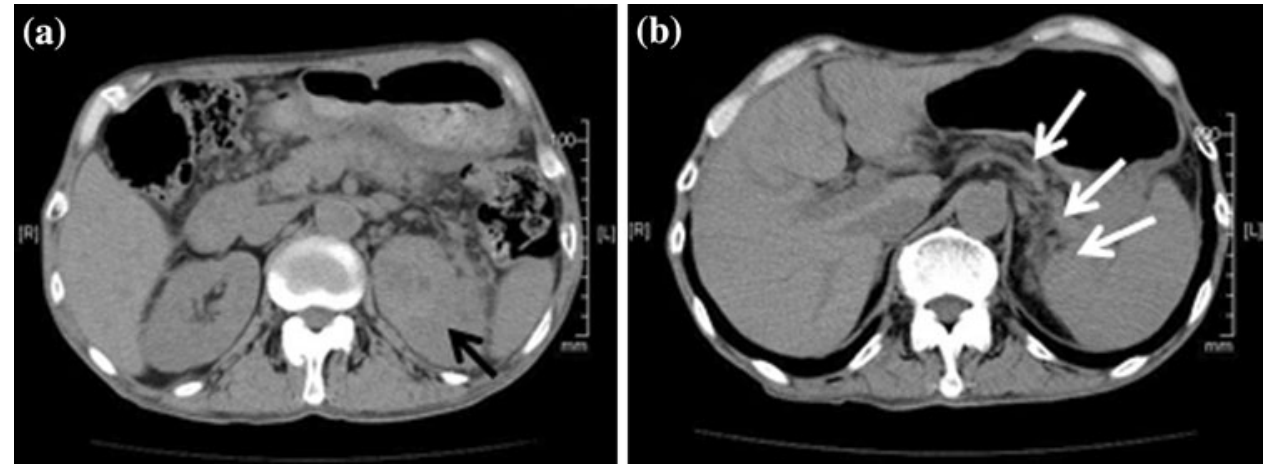

At that time, the patient seemed not to be a candidate for cytoreductive nephrectomy because of the possible diagnosis of peritoneal carcinomatosis and his poor general condition. We treated the patient with sorafenib (Nexa$\operatorname{var}^{\circledR}$ ) $800 \mathrm{mg}$ daily. After 4 weeks of treatment with sorafenib, grade 3 thrombocytopenia (according to the National Cancer Institute/Common Toxicity Criteria for Adverse Events version 3.0) was observed, which improved after reduction of the sorafenib dose to $400 \mathrm{mg}$. After 3 months of treatment with sorafenib, a CT scan showed a stable left renal mass and the peritoneal nodules; however, the ascites had disappeared (Fig. 2). Therefore, confidence in the diagnosis of peritoneal carcinomatosis was clinically obtained. After 6 months of treatment with sorafenib, the therapy was withdrawn owing to acute pancreatitis because of a pancreatic duct stone and grade 3 renal dysfunction. A follow-up CT scan after 3 months of no sorafenib therapy showed a stable renal mass and no ascites with $95 \%$ reduction in the peritoneal nodules (Fig. 3). After 6 months of no sorafenib therapy, the patient's acute pancreatitis was improved after the placement of a pancreatic duct stent, and renal dysfunction recovered to the grade 1 level. Repeat imaging still showed stable left renal mass and peritoneal nodules. Concerns about his quality of life on dialysis led the patient to avoid nephrectomy. Sorafenib treatment was resumed at a dose of $400 \mathrm{mg}$ every other day.

\section{Discussion}

The treatment for metastatic renal cell carcinoma (mRCC) has changed dramatically since the introduction of molecular-targeted therapies including tyrosine kinase 
inhibitors. These tyrosine kinase inhibitors have been shown to reduce tumors and prolong survival of patients with $\mathrm{mRCC}$ when compared with cytokine therapy [3, 4]. They also induce a high partial response rate at metastatic sites, with the most common sites of response being the lungs and lymph nodes. Peritoneal carcinomatosis is a relatively rare presentation of RCC. RCC accounts for only $2 \%$ of primary neoplasms that metastasize to the peritoneum [1]. Although the ascitic fluid cytology exam was negative in this case, we nevertheless suspected a malignant etiology from the characteristics of the ascitic fluid because the vast majority $(82.8 \%)$ of patients have a SAAG of less than 1.1, and cytological examination of ascitic fluid is highly specific and its diagnostic sensitivity is only about $40-60 \%$ [5].

VEGF-targeted molecular therapy has been reported to inhibit angiogenesis and tumor growth. VEGF is also known as a tumor-secreted permeability factor, and has been reported in ascitic fluid accumulation [6]. Blockade of VEGF using monoclonal antibodies or low molecular weight inhibitors has been shown previously to dramatically reduce ascites formation and tumor burden in peritoneal tumor models [7, 8]. Therefore, tyrosine kinase inhibitors should be chosen as a first-line therapy in advanced RCC patients with peritoneal carcinomatosis with ascites.

This is the first reported case of a therapeutic response to sorafenib by an advanced RCC patient with peritoneal carcinomatosis. This patient has had longer than 15 months of control of the mRCC with an unresected renal primary tumor following the target therapy. Relatively few data are available to indicate which patients might benefit from cytoreductive nephrectomy. However, the ongoing improvement in this patient's peritoneal nodules and ascites and the stability of the renal mass have encouraged us to continue treatment for patients who are not candidates for cytoreductive nephrectomy because of their poor general condition and renal insufficiency.

Conflict of interest There is no financial or commercial interest in the paper.

\section{References}

1. Tartar VM, Heiken JP, McClennan BL (1991) Renal cell carcinoma presenting with diffuse peritoneal metastases: CT findings. J Comput Assist Tomogr 15:450-453

2. Rini BI, Flaherty K (2008) Clinical effect and future considerations for molecularly-targeted therapy in renal cell carcinoma. Urol Oncol 26:543-549

3. Escudier B, Szczylik C, Hutson TE et al (2009) Randomized phase II trial of first-line treatment with sorafenib versus interferon alfa$2 \mathrm{a}$ in patients with metastatic renal cell carcinoma. J Clin Oncol 10:1280-1289

4. Motzer RJ, Hutson TE, Tomczak P et al (2007) Sunitinib versus interferon alfa in metastatic renal-cell carcinoma. N Engl J Med 11:115-124

5. Tuzun Y, Yilmaz S, Dursun M et al (2009) How to increase the diagnostic value of malignancy-related ascites: discriminative ability of the ascitic tumour markers. J Int Med Res 37:87-95

6. Senger DR, Galli SJ, Dvorak HF et al (1983) Tumor cells secrete a vascular permeability factor that promotes accumulation of ascites fluid. Science 219:983-985

7. Shaheen RM, Ahmad SA, Ellis LM et al (2001) Inhibited growth of colon cancer carcinomatosis by antibodies to vascular endothelial and epidermal growth factor receptors. $\mathrm{Br} \mathrm{J}$ Cancer 85:584-589

8. Machida S, Saga Y, Suzuki M et al (2005) Inhibition of peritoneal dissemination of ovarian cancer by tyrosine kinase receptor inhibitor SU6668 (TSU-68). Int J Cancer 114:224-229 